\title{
THE NEED FOR TRIGGERING IN COLD FUSION REACTIONS
}

\author{
M. MCKUBRE, F. TANZELLA \\ SRI International, Menlo Park, CA \\ P. HAGELSTEIN \\ MIT Cambridge, $M A$ \\ K. MULLICAN AND M. TREVITHICK \\ Project Cobalt, Sunnyvale, $C A$
}

\section{Introduction}

It has long been recognized that initiation of the cold fusion heat effect observed in heavy water electrolysis at palladium cathodes requires simultaneous attainment of three conditions: (i) high loading or chemical potential of D within the Pd lattice; (ii) an initiation time at least ten times larger than the D diffusion time constant: (iii) a minimum or threshold electrochemical surface current or current density that is not correlated to the bulk D loading [1-4]. In 1995 a fourth condition was added with the recognition [5] that (iv) deuterium flux plays an important role in determining the excess heat power density.

With the emergence of better phenomenological understanding and predictive theory we can begin to associate the features of the observed causes with the requirements of a solid state nuclear effect that gives rise to heat and associated He-4. We understand that the production of significant excess heat from a solid target requires thermodynamic preconditioning (i and ii) and suitable triggering (iii and iv). Electrochemistry provides a convenient means of loading but it is energetically expensive to maintain the electrochemical current density just to preserve loading during the initiation period if alternative means are available. More importantly, triggering by means of a high-density electrochemical current flux is an inefficient way to achieve the favorable energy balance necessary to demonstrate commercial potential of the effect. In this paper we will review briefly the history that has lead us to our present understanding, and explore means to separate the various steps necessary for excess heat production in bulk palladium or other suitable host materials. In particular we are concerned with decoupling the deuterium loading from the nuclear triggering processes as a pathway towards practical application of the cold fusion effect. We report on the results of one experiment, also reported by an-other group $[6,7]$ at this conference, apparently confirming that a deuterium loaded palladium cathode can be triggered by non-electrochemical means to produce new or increased excess heat production rates. 


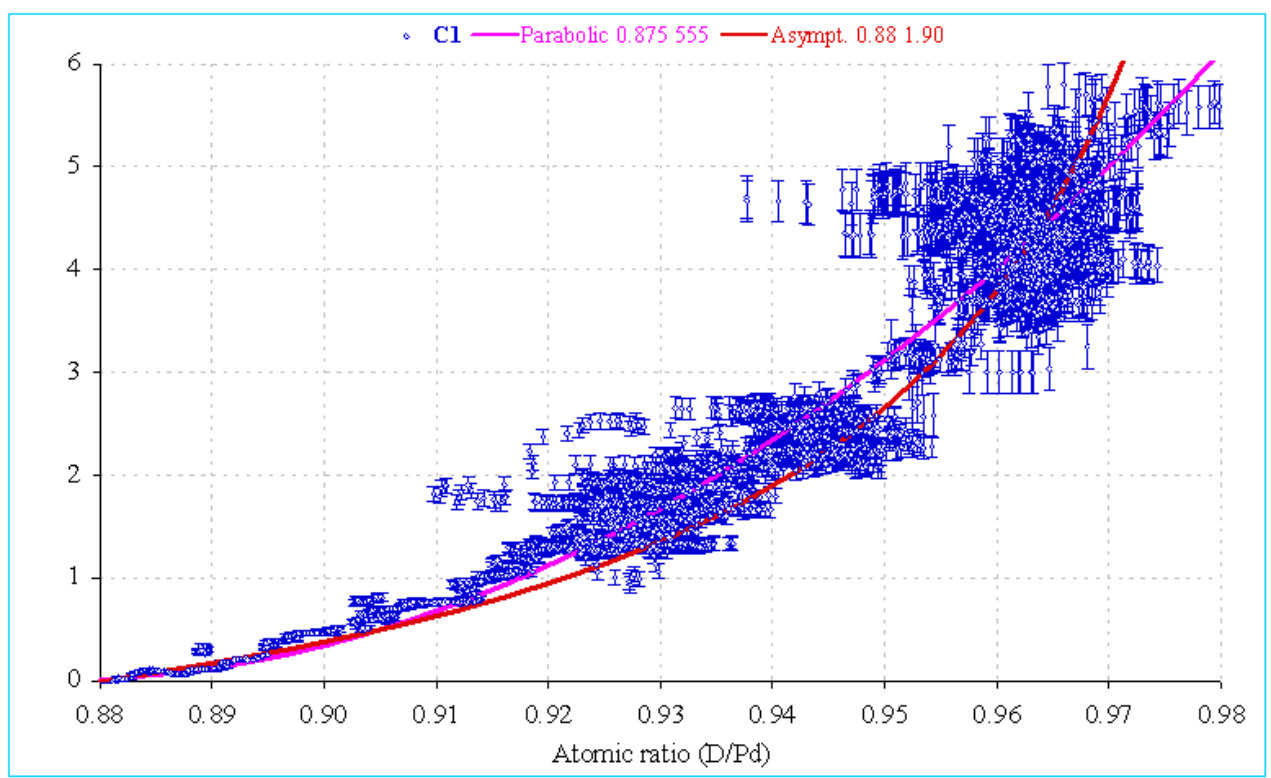

Figure 1. Excess power density in $W / \mathrm{cm} 3$ versus average $D / P d$ atomic ratio measured from the axial resistance for a Johnson Matthey wire cathode $30 \mathrm{~cm}$ long and $1 \mathrm{~mm}$ in diameter in $1.0 \mathrm{M}$ LiOD containing 200 ppm Al.

\section{History}

The hypothesis that guided early studies of the putative "cold fusion" effect was that: "There is an unexpected and unexplained source of heat in the D/Pd system that may be observed when deuterium is loaded electrochemically into the palladium lattice to a sufficient degree." Experimental verification of this hypothesis and quantification of the effect required a considerable investment of research funds and time because of the need to establish:

- $\quad$ Accurate methods of measuring the deuterium loading in palladium cathodes (the $\mathrm{D} / \mathrm{Pd}$ atomic ration), reliably, in-situ and in real time.

- $\quad$ Advanced electrochemical procedures to load deuterium into palladium to hitherto unimagined high levels in aqueous systems.

- $\quad$ Reliable, accurate and stable calorimetric methods to measure heat evolved at small fractional rates in excess of the electrical input power, and chemical and mechanical input energies.

These combined technical issues were challenging. It was not until ICCF-3 in Nagoya, Japan, October 1992, over 3 years after the Fleischmann Pons announcement in March 1989, that clear definition was made of the functional relationship between D/Pd loading and (volumetric) excess heat production rates simultaneously by two groups, one at IMRA Japan[3] and the other at SRI[4], whose results are reproduced here as Figure 1. 


\subsection{Excess heat and PdD loading}

In the following years it became clear that necessary conditions for excess heat production in palladium were cathodes loaded electrolytically with deuterium included:

- $\quad$ Maintenance of a high average $\mathrm{D} / \mathrm{Pd}$ ratio (loading)

- For times between 20 and 50 times longer than are needed for deuterium diffusion into the lattice (initiation)

- At electrolytic current densities greater than $250-500 \mathrm{~mA} \mathrm{~cm}^{-2}$ (stimulation).

Each of these requirements needs further discussion. The minimum average loading threshold indicated in Figure 1 is $\mathrm{D} / \mathrm{Pd} \geq 0.875$ and values on the order of $0.84-0.88$ have typically been observed. Nothing special happens in the $\mathrm{Pd} / \mathrm{D}$ phase diagram in this compositional range; neither do we expect the composition to be homogeneous throughout the Pd electrode in the strongly disequilibrium conditions imposed by the superficial cathodic current flux. One thought is that high average bulk loading is needed to permit the nucleation and maintenance of special zones having higher stoichiometries or chemical potential near or at the cathode surface. Several "special" compositions have been suggested although not fully specified or directly observed. Two involve a conjectured "gamma" phase of the Pd/D system: one resulting from tetrahedral site occupancy of the Pd fcc lattice; the other from double occupation of octahedral sites. A special site called by Storms [8] "nuclear active" is confined to the surface and may involve electrolyte-derived elements in addition to Pd and D. Whatever the case, "loading" should be viewed as an indirect and lower bound diagnostic of a more precise metallurgical and/or chemical condition needed to promote the "cold fusion" effect.

The need to maintain high loading for long times following its (sometimes maximal) attainment also requires further comment. Cathodes exercised at high current densities and loadings greater than the threshold value but times shorter than the critical "initiation" time a were not observed in at SRI to produce measurable levels of excess heat. Some process other than deuterium diffusion and loading into the lattice is needed for excess heat production.

\subsection{Threshold in electrochemical current density}

The requirement for high electrochemical currents or current densities also is largely unexplained. The fact of this requirement was clearly recognized by Fleischmann and Pons in their early publications $[9,10]$ and has been verified in numerous laboratories $[3,4,11]$. What is not widely recognized is that these threshold electrochemical densities far exceed those needed for the attainment of maximum loading. In LiOD electrolyte the highest levels of electrochemical loading can be obtained between 10 and $20 \mathrm{~mA} \mathrm{~cm}$. The equilibrium loading slowly decreases at higher current densities as the cathodic surface becomes screened by bubbles, and damaged and contaminated from the high rate of electrochemical work. Thus, as for the initiation effect, the electrochemical action on the surface plays a role in producing an excess heat effect, over and above that of discharging $\mathrm{D}$ atoms at the cathode surface.

\footnotetext{
${ }^{\mathrm{a}}$ Initiation times for $1 \mathrm{~mm}$ diameter wires were $\sim 160-190$ hours and for $3 \mathrm{~mm}$ diameter wires $\sim 300-500$ hours. Full and sometimes maximum loading is obtained by electrochemical means for $1 \mathrm{~mm}$ wires in about 8-12 hours, and $3 \mathrm{~mm}$ wires in about 24-36 hours.
} 


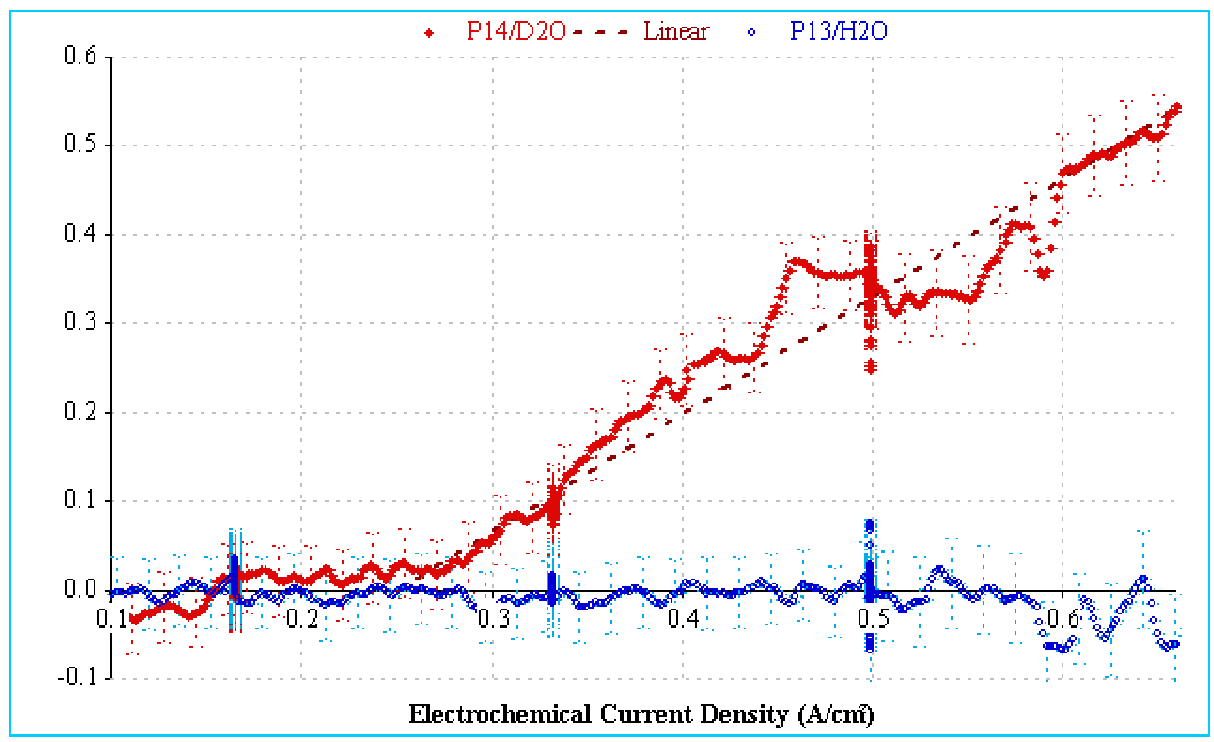

Figure 2. Excess power as a function of current density in twin calorimeters and cells containing $3 \mathrm{~cm}$ long 3 mm diameter Engelhard Pd cathodes in 1.0 M LiOH (blue) and LiOD (red).

The functional relationship of excess power with electrochemical current is very closely linear above a non-zero threshold. Figure 2 shows an early and typical result measured at SRI[2] and Storms has reviewed numerous examples [11]. In the discussion we will consider further the causes of this effect and why it is of engineering significance.

\subsection{Excess heat and interfacial deuterium flux}

In 1991 Bockris et al [12] first commented on the relationship between excess heat and interfacial deuterium flux. This was further discussed in 1995 as a criterion for the existence of an excess heat effect in Pd wire cathodes [5]. For experiments in which both excess heat and quantitatively correlated ${ }^{4} \mathrm{He}$ was measured, a functional relationship was established between the rate of excess heat production and the rate of deuterium compositional fluctuation within the cathode. Since the average bulk composition can only vary as a flux of deuterium through the surface, this last was interpreted to mean that the excess power effect is correlated with (as either cause or effect of) a deuterium flux at the interface. 


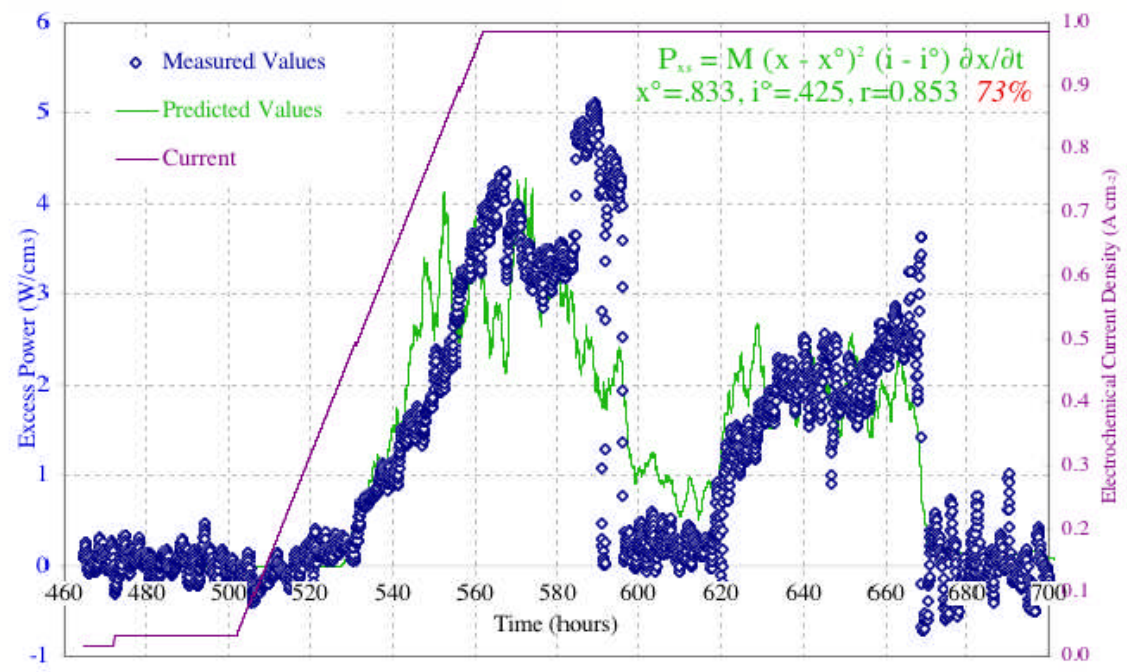

Figure 3. Excess power as a function of time and fitting function for experiment M4 using a Johnson Matthey Pd wire cathode $10 \mathrm{~cm}$ long and $1 \mathrm{~mm}$ diameter in $1.0 \mathrm{M}$ LiOD containing $200 \mathrm{ppm} \mathrm{Al}$.

Figure 3 presents the results of an experiment performed at SRI designated as M4 in which a $1 \mathrm{~mm}$ diameter, $10 \mathrm{~cm}$ length of high purity Johnson Matthey Pd wire was electrolyzed in $1 \mathrm{M}$ LiOD inside a helium leak-tight, metal-sealed, PTFE-lined cell, contained within a high accuracy mass-flow calorimeter [13]. The data in Figure 3 show the second occasion in which an attempt was made using a current ramp (the solid line referenced to the right is in Figure 3) to stimulate the excess heat effect. The first attempt at 200 hours resulted in no measured departure from thermal equilibrium in the calorimeter.

Several features are evident from Figure 3 and subsequent analyses:

- Initiation of excess power (open diamonds referenced to the left axis) occurs 30 hours after the initiation of the current ramp at a (rather large) critical current density of 425 $\mathrm{mA} \mathrm{cm}{ }^{-2}$.

- $\quad$ Excess power initially depends linearly on current or current density above this threshold value and sustains with some variability at the maximum electrochemical current.

- $\quad$ Approximately 32 hours after cessation of the current ramp, while all system variables were being controlled at constant values, the excess power dropped to near zero which value was maintained for over 20 hours.

- $\quad$ Production of excess power resumed, increased and then terminally subsided, all apparently spontaneously under intentionally constant input conditions.

- $\quad$ From post-test analyses of the gaseous contents of the cell the amount of 4He evolved was found to correlate closely $(105 \pm 10 \%)$ with the integral of excess power $[5,13]$ assuming the reaction $\mathrm{D}+\mathrm{D} \rightarrow{ }^{4} \mathrm{He}+23.8 \mathrm{MeV}_{\text {lattice. }}$

Some clarification of the apparently uncontrolled magnitude of excess power can be obtained by inspecting the behavior of the cathode loading. Figure 4 shows the response of the $\mathrm{D} / \mathrm{Pd}$ ratio to the applied current ramp in the time interval shown in Figure 3. 


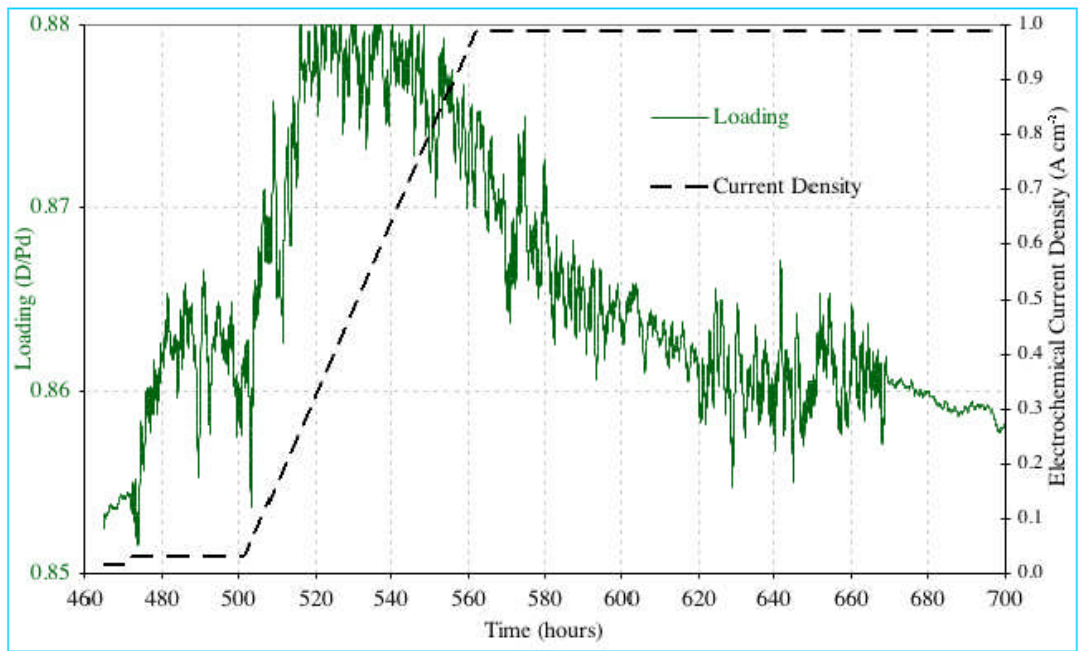

Figure 4. Electrochemical current density and loading for experiment M4.

Several features of Figure 4 are of particular significance:

- Initially, the loading rises with applied electrochemical current density, reaching its maximum value at almost the time and current density at which the excess power excursion begins.

- $\quad$ The mean loading value falls monotonically with time from that point, both with current increasing and constant.

- $\quad$ The time record of the cathode bulk average loading (measured from the axial resistance ratio) shows a high transient variability about its mean value. This variation is not noise but the signal of a cyclic loading/unloading process.

- $\quad$ The amplitude of the loading/unloading cycle appears to correlate with the magnitude of the excess power.

The characteristics of the loading/unloading cycle are more clearly seen in Figure 5 which shows an expansion of the data in Figures 3 and 4 encompassing the region of the (apparently spontaneous) re-initiation of the excess power effect at $\sim 618$ hours. An approximately sinusoidal cycle can be seen within a period $\sim 2$ hours and amplitude that increases abruptly at the time of excess power re-initiation. 


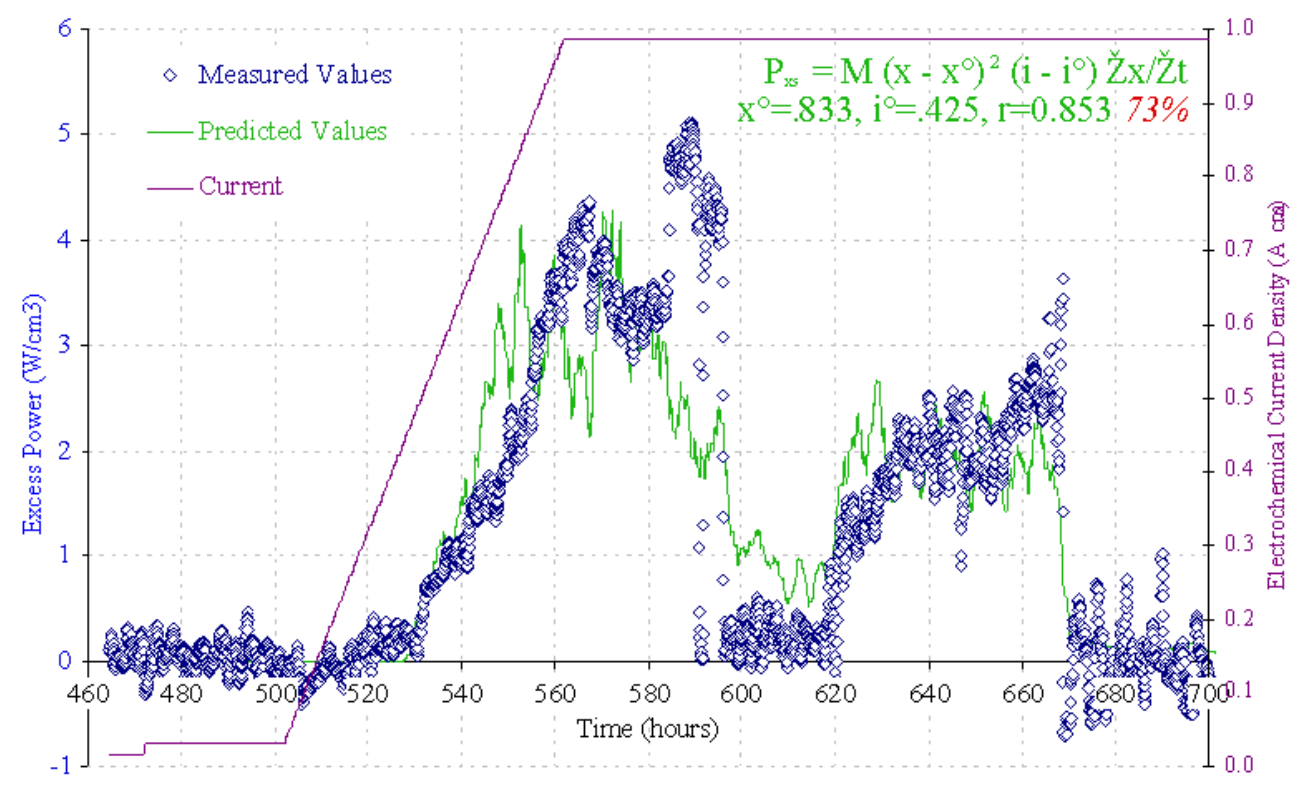

Figure 5. Excess power and loading for experiment M4.

This cyclic change in bulk, average loading results from a flux or "breathing" of deuterium atoms in and out of the metal cathode through its surface. The origins of this periodic phenomenon are as yet unexplained, as are the reasons for its change in amplitude (i.e. rate). The cyclic period can at least partially be understood from the natural time constant of diffusion. If we substitute the cathode radius $r=0.05 \mathrm{~cm}$ and period $\mathrm{r}=7200 \mathrm{~s}$ into the simple expression for the advance of a diffusion front, $r=\sqrt{ } 2 \mathrm{Dt}$, we obtain for $\mathrm{D}$ a value of $3.5 \times 10^{-7} \mathrm{~cm}^{2} \mathrm{~s}^{-1}$, about twice that for the diffusion of $\mathrm{D}$ in $\mathrm{PdD}$ at $30^{\circ} \mathrm{C}$. This fundamental frequency was confirmed for one other cathode of $1 \mathrm{~mm}$ diameter that successfully produced excess heat at SRI $[5,13]$. The cyclic "breathing" phenomenon was not confirmed in $3 \mathrm{~mm}$ and larger diameter cathodes possibly because of the following technical difficulties:

- $\quad$ Loading was inferred from measured resistance. Resistance decreases markedly as diameter is increased and length decreased for the same surface area or volume of metal.

- $\quad$ The time constant of diffusion increases with the square of radius.

- The rate at which the deuterium "reservoir" in the cathode can be filled and unfilled is governed by the ratio of surface to volume, which decreases strongly with cathode length.

Whatever the reason or reasons for its non-observance in $3 \mathrm{~mm}$ and larger diameter cathodes, it is clear that the magnitude of the excess heat effect observed in $1 \mathrm{~mm}$ diameter cathodes correlates closely with the rate of progress of a diffusion front of $\mathrm{D}$ atoms entering the surface and progressing to the cathode center, and with the reverse process of deloading that results from collision of diffusion fronts and overlapping from opposite sides.

\section{Discussion}

The results reviewed above suggest that, in addition to attaining and maintaining high static average loadings of $\mathrm{D}$ in $\mathrm{Pd}$, you need motion relative to the lattice to stimulate nuclear effects in 
electrolytically loaded Pd wire cathodes. We have discussed this effect as the need for "triggering", and the required motion may take one or more of several forms:

- Motion of the "host" (Pd or other) atoms relative to the lattice. We exclude from consideration here the diffusion of lattice vacancies which we consider as an initiation processes. Included are all forms of lattice vibration (although coherent modes are likely to have more effect).

- The motion of interstitial "guest" atoms (D) through the lattice especially the effect of causing fluxing D atoms to transit a steep gradient in chemical potential (e.g. at the surface or in a deliberately layered structure). This motion results from imposed chemical and/or electrical potential gradients.

- $\quad$ The flow of electrons through the metal in the radial direction due to electrochemical discharge of $\mathrm{D}+$ at the cathode surface in the axial direction due to an imposed electrical potential gradient along the wire.

In electrochemical experiments we propose that one or more of these effects result from electrochemical discharge and adsorption/desorption of $\mathrm{D}$ at the cathodic surface. We can write two generic equations for this process for electrochemical reactions performed in acid or basic electrolytes:

$$
\begin{aligned}
& \mathrm{D}^{+}+\mathrm{e}^{-} \leftrightarrow \mathrm{D}_{a d s} \leftrightarrow \mathrm{D}_{a b s} \quad \text { (acid) } \\
& \mathrm{D}_{2} \mathrm{O}+\mathrm{e}^{-} \leftrightarrow \mathrm{OD}^{-}+\mathrm{D}_{a d s} \leftrightarrow \mathrm{D}_{a b s} \quad \text { (base) }
\end{aligned}
$$

Either of these reactions can, when properly performed, cause high bulk loading by driving the first equilibrium far to the right to produce $\mathrm{D}_{a d s}$ species at very high chemical potential. These reactions also are capable of providing the triggering in one of three modes:

- A high current density normal to the surface associated with the process of electron transfer from the metal to a surface-localized $\mathrm{D}_{2} \mathrm{O}$ molecule or $\mathrm{D}^{+}$ion.

- $\quad$ A cyclic adsorption/desorption process of the sort demonstrated in Figures 4 and 5 caused by periodic alternation of the relative chemical potentials of $\mathrm{D}_{a d s}$ and $\mathrm{D}_{a b s}$.

- $\quad$ A displaced exchange flux in which $\mathrm{D}$ atoms are deposited and adsorbed preferentially at one site and desorbed and then evolved as $\mathrm{D}_{2}$ molecules at a second site somewhat removed.

A major contention of this paper is that electrochemistry may very well provide an ideal means of loading D into Pd, but suffers from a number of practical limitations when required also to provide the triggering. Principal among these limitations is that sustaining the electrochemical process long after maximum loading is obtained is energetically highly inefficient and potentially hazardous. The (heavy) water molecule must cleave to form $\mathrm{D}_{a d s}$ which process requires consider-able investment of voltage (a minimum of $1.27 \mathrm{~V}$ ) and therefore energy. This energy must be accounted for in the denominator of any power or energy gain calculation.

Once stable loading is obtained, all of the $\mathrm{D}$ atoms presented to the surface recombine to form $\mathrm{D}_{2}$ which then bubbles up from the cathode surface to join $\mathrm{O}_{2}$ in the headspace. In practical applications this nearly stoichiometric gas mixture cannot be released because of the cost, and is not easily recombined in a guaranteed safe manner. 
It is not at all clear how effectively the electron charge transfer reaction or the adsorption/adsorption reaction couple energy into modes of lattice vibration appropriate to stimulate D + D interaction. The fundamental electrochemical process involving the transfer of an electron from the conduction band of a metallic electrode to or from a molecule ion is not fast on the scale of lattice vibration.

In order to provide a suitable flux of D through the cathode surface one cannot sustain either net loading or unloading: the first because $\mathrm{Pd}$ has a bulk absorption limit of $\sim \mathrm{PdD}_{1.0}$; the second because the excess heat effect exhibits a minimum threshold of $\sim \mathrm{PdD}_{0.85}$. Instead one must rely on either an alternating loading/unloading process or an exchange flux of balanced absorption and desorption at separated sites. The conditions under which one can reliably produce either of these effects have yet to be defined, and may have been achieved in previous experiments only adventitiously.

From this discussion, emerge the following interim conclusions. In order to increase the probability of making practical use of the heat effect produced by D in Pd electrodes, the necessary steps of loading, initiation, triggering must be decoupled. Electrochemistry provides a suitable, possibly ideal pathway to load $\mathrm{D}$ at very high chemical potential into $\mathrm{Pd}$. If the loading can be sealed, with previous, simultaneous or subsequent processing, then alternative means of triggering can be employed.

As discussed above deuterium flux and electron flux have both been used successfully to trigger nuclear heat release. Unfortunately both were very inefficient requiring large amounts of energy Input. Some authors have reported success with acoustic stimulation, but the coupling of input energy into the needed modes of (lattice) stimulation also appears to be very inefficient. The need for triggering was recognized early by Bockris et al [14] and radio frequency electromagnetic stimulation was attempted with some success. Ultraviolet stimulation was proposed by Bass [15], but not attempted. Ideally what is sought is a standard, commercial, controllable, coherent source that is small, inexpensive and easily coupled to the experiment or intended heat source.

\section{Experiments On Laser Triggering}

Solid-state red lasers appear to meet the criteria of an "ideal" triggering source and are available and controllable in a range of wavelengths. They are also relatively inexpensive, readily available and have been demonstrated in at least one experimental program to effectively trigger the excess heat effect in palladium cathode loaded electrolytically with deuterium [6].

In an attempt to demonstrate that a laser trigger effect could be decoupled from the electrochemical loading and triggering effects, experiments were performed following the protocols outlined and supported by D. Letts [6, 7]. Cathodes constructed of 7 x 5 x $0.25 \mathrm{~mm} \mathrm{Pd}$ foils prepared and mechanically treated by Letts were electrolyzed in $0.1 \mathrm{M} \mathrm{LiOD}$ prepared from Alfa Aesar $99.9 \%$ pure Li foil dissolved in $\mathrm{D}_{2} \mathrm{O}$ sourced from Sigma Aldrich. Experiments were performed in a $250 \mathrm{ml}$ Pyrex vessel equipped with a closely fitting PTFE lid with holes to accommodate: anode and cathode electrical contacts; contact to a secondary sacrificial Au anode; calorimetric and electrolyte temperature sensors; water make-up and a gas over-pressure relief tube.

The Pd cathodes were spot-welded to $10 \mathrm{~cm}$ of $1 \mathrm{~mm}$ diameter Pt lead wire. The primary anode was constructed from $30 \mathrm{~cm}$ of $0.5 \mathrm{~mm}$ diameter Pt configured as a vertical helix 
symmetrically surrounding the cathode and extending $1 \mathrm{~cm}$ above and below the cathode to assure approximately uniform current distribution. A secondary anode constructed of a $5 \times 5 \times$ $0.25 \mathrm{~mm} \mathrm{Au}$ foil spot-welded to $10 \mathrm{~cm}$ of $1 \mathrm{~mm}$ diameter Pt wire was included to allow Au to be electrochemically transferred to the cathode.

An initial current density of $0.7 \mathrm{~mA} \mathrm{~cm}^{-2}$ was applied for a period of 7 days to load $\mathrm{D}$ into the Pd cathode without fracturing the lattice. Current was increased over a period of 14 hours to 12 $\mathrm{mA} \mathrm{cm}{ }^{-2}$ once a sufficient loading ratio had been reached. For the remaining duration of the experiment, the system was operated in a constant power mode at $\mathrm{M}$. If, during the course of the experiment, the current density fell below $1 \mathrm{~mA} \mathrm{~cm}^{-2}$, the power was increased.

Calorimetry was performed in isoperibolic mode by measuring the temperature difference between calibrated thermistors placed in the electrolyte and the surrounding constant temperature enclosure. In the conditions of electrolysis under which calorimetric results are reported here, the electrolyte contents of the cell were well stirred, and the isoperibolic calorimetry constant was determined as $\mathrm{K}=0.240 \pm 0.002 \mathrm{~W} \mathrm{~K}^{-1}$.

A $680 \mathrm{~nm}$ solid-state laser was positioned with beam closely orthogonal to one surface of the foil cathode. The total path length of the beam was $\sim 100 \mathrm{~cm}$, passing through the double glass front door of the thermal enclosure, the Pyrex cell wall, and $\sim 3 \mathrm{~cm}$ of electrolyte. The wavelength and intensity of the laser were controlled using an ILX Lightwave LDC-3700B controller driving an ILX Lightwave LDM-4407 laser mount. By comparing the power registered calorimetrically with that measured on a StellarNet EPP2000 spectrometer, it was determined that $\sim 57 \%$ (17 of $30 \mathrm{~mW}$ ) of source laser power was coupled into the electrochemical cell, the rest being reflected or absorbed outside the cell in the range of wavelengths employed.

\subsection{Results}

Experiments were performed to test the effects of two variables in triggering a laser excess heat effect: the presence or absence of laser illumination and wavelength in the range 679-686 nm; and the presence and degree of Au deposit on the electrode surface. Figure 6 shows the results of one such experiment after 1 day of cathode loading. The solid line and error bars are excess power computed as output power $\left(=\mathrm{K} \Delta \mathrm{T}_{\text {in-out }}\right)$ minus electrochemical input power minus 0.57 times laser input power. These values are referred to the left is.

The trapezoidal dashed lines at the bottom of Figure 6 plot the laser wavelength that was swept linearly from 679 to $686 \mathrm{~nm}$ for 6 hours followed by 6 hours of no illumination. This process was repeated for the duration of the experiment. As can clearly be seen in Figure 6 the first 4 cycles of laser illumination and wavelength ramping resulted in no observable departure from thermal equilibrium with all data falling inside the assigned error bounds. 


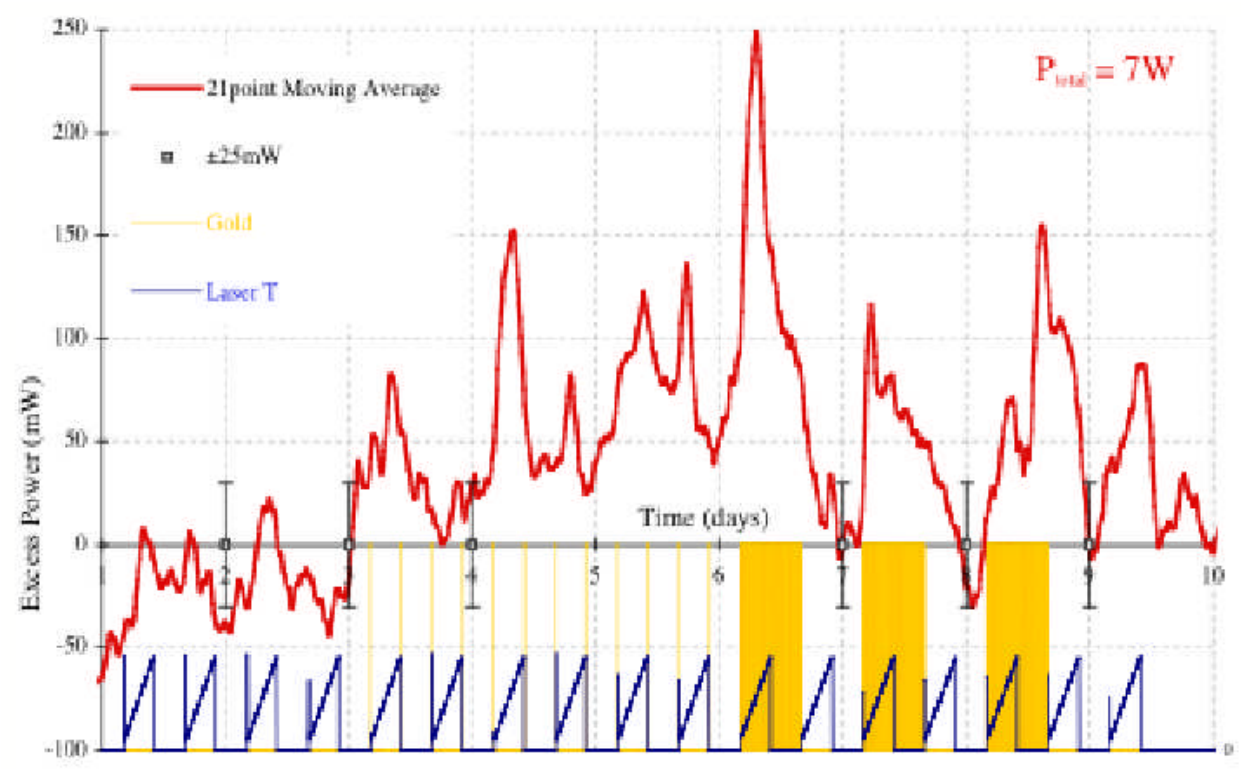

Figure 6. The effects of laser stimulation and Au deposition on the excess Power produced by a $7 \times 5 \times 0.25$ mm Pd foil cathode in $0.1 \mathrm{M} \mathrm{LiOD.}$

The vertical spike first appearing at 3.2 days represents the application of positive potential to the Au secondary anode in an attempt to dissolve gold in the electrolyte and allow it to transfer to the Pd cathode. This process is not well quantified, and the units used to plot it in Figure 6 are arbitrary. Initially an Au anode was simply connected electrically to the primary anode for 10 minutes every 6 hours. The result of this process was a discernable blackening of the cathode surface as well as black sediment appearing at the bottom on the chamber.

The data in Figure 6 show that the first application of Au conjointly with laser stimulation resulted in a significant increase in the measured excess power. Perhaps equally significantly, when the second pulse of Au was applied without subsequent laser illumination, then excess power did not increase and, instead, declined towards the thermal equilibrium value. Close inspection of these data reveals a pattern of increasing $\mathrm{P}_{x s}$ following Au deposition with the laser on, but decreasing with the laser off, appears generally in the data. This suggests that both laser illumination and Au deposits are necessary to produce the effect of laser stimulated excess heat. In no case was laser stimulated heating observed in the absence of a deliberately introduced specular modification of the Pd cathode surface.

The data in Figure 6 are too scattered, and the time constants of response too long to uncover any wavelength dependence of the laser excess heating effect. Attempts to correlate either the absolute value of the measured excess power or its time derivative, with laser wavelength, produced results having no statistical significance.

After 6.2 days an attempt was made, apparently successfully, to increase a modest excess power in the range $50-100 \mathrm{~mW}$, by forming a more substantial gold deposit. Instead of a single 10 minute anodization at the beginning and end of the laser sweep cycle, the Au electrode was switched on for 12 hours in conjunction with a 6 hour laser sweep and the subsequent 6 hour laser off period, and then switched off for 12 hours. The result was a rapid increase in $\mathrm{P}_{x s}$ to a maximum value $>300 \mathrm{~mW}$ in the presence of laser illumination, followed by an equally rapid 
decline which started even before the laser was switched off. The subsequent sweep of laser illumination resulted in a further decline of $\mathrm{P}_{x s}$.

Another attempt was made at 7.2 days to repeat the beneficial effects of "massive" Au deposition. As can be seen in Figure 6 this resulted in a rapid but small increase in $\mathrm{P}_{x s}$ followed by a prolonged decline to thermal equilibrium. From these results we conclude simply that laser illumination can effectively stimulate an excess heat effect in a Pd cathode previously loaded and maintained loaded with D electrochemically, but only when the surface is modified. In our case the modification was Au metal, electrodeposited from solution with sufficiently small dimension to appear black. Increasing the extent of deposit increases the effect, up to a certain point, after which the surface is masked or modified in such a way as to prevent the laser stimulated excess power effect.

\section{Conclusions}

Preliminary results suggest the possibility of decoupling the needs of chemical pre-conditioning and disequilibrium stimulus to trigger the Fleischmann Pons excess heat effect. Discovery and development of effective means to seal or otherwise stabilize the required material configuration and to stimulate required lattice interactions will allow the reproducible attainment of the effect and intensive scale-up of power gain. Both are pre-conditions to the development of full scientific understanding and for possible practical application.

\section{Acknowledgements}

The authors acknowledge the technical support and intellectual guidance of Dennis Letts and Dennis Cravens in the laser stimulation studies reported here.

\section{References}

1. M.C.H. McKubre, R.C. Rocha-Filho, S. I. Smedley and F. L. Tanzella, "Calorimetry and Electrochemistry in the D/Pd System", Proceedings of the 1st International Conference on Cold Fusion, Mar. 1990 Salt Lake City, UT, edited by F. Will, p. 20.

2. M. C. H. McKubre, R. Rocha-Filho, S. I. Smedley, F. L. Tanzella, S. Crouch-Baker, T. O. Passell, and J. Santucci, The Science of Cold Fusion, Proc. of the Second Annual Conference on Cold Fusion, Como, Italy, June 29-July 4, 1991, edited by T. Bressani, E. Del Giudice and G. Preparata, Published by Societa Italiana di Fisica, 33, p. 419.

3. M.C.H. McKubre, S. Crouch-Baker, A. M. Riley, S. I. Smedley and F. L. Tanzella, "Excess power observations in electrochemical studies of the D/Pd system; the influence of loading" Frontiers of Cold Fusion, Proceedings of the 3rd International Conference on Cold Fusion, Dec. 1993 Nagoya, Japan, edited by H. Ikegami, Universal Academy Press, Tokyo, p. 5.

4. K. Kunimatsu, N. Hasegawa, A. Kabota, N. Imai, N. Ishikawa, H. Akita and Y. Tsuchida, "Deuterium Loading Ratio and Excess Heat Generation during Electrolysis of Heavy Water by a Palladium Cathode in a Closed Cell Using a Partially Immersed Fuel Cell Anode," Frontiers of Cold Fusion, Proceedings of the 3rd International Conference on Cold Fusion, Dec. 1993 Nagoya, Japan, edited by H. Ikegami, Universal Academy Press, Tokyo, p. 31.

5. M.C.H. McKubre, S. Crouch-Baker, A.K. Hauser, S.I. Smedley, F.L. Tanzella, M.S. Williams, and S.S. Wing, "Concerning Reproducibility of Excess Power Production", Proceedings of the 5th International Conference on Cold Fusion, Monte Carlo, Monaco April 9-13, 1995, IMRA Europe, Sophia Antipolis Cedex, France (1995), p. 17. 
6. 6. D. Letts and D. Cravens, "Laser stimulation of deuterated palladium: Past and present," Proceedings of the 10th International Conference on Cold Fusion, August 2003 Cambridge, MA, edited by P. L. Hagelstein and S. R. Chubb, World Scientific, to appear.

7. D. Cravens and D. Letts, "Practical techniques in CF research: Triggering methods," Proceedings of the 10th International Conference on Cold Fusion, August 2003 Cambridge, MA, edited by P. L. Hagelstein and S. R. Chubb, World Scientific, to appear.

8. E. K. Storms, "Some Thoughts on the Nature of the Nuclear-Active Regions in Palladium", in Proceedings of the 6th International Conference on Cold Fusion, October 1996 Lake Toya, Japan, edited by M. Okamoto.

9. M. Fleischmann, S. Pons and M. Hawkins, "Calorimetry of the Palladium-Deuterium System," Proceedings of the 1st International Conference on Cold Fusion, Mar. 1990 Salt Lake City, UT, edited by F. Will, p. 1.

10. S. Pons and M. Fleischmann, The Science of Cold Fusion, Proc. of the Second Annual Conference on Cold Fusion, Como, Italy, June 29-July 4, 1991, edited by T. Bressani, E. Del Giudice and G. Preparata, Published by Societa Italiana di Fisica, p. 349.

11. E. K. Storms, "Measurement of Excess Heat from a Pons-Fleischmann Type Electrolytic Cell," Frontiers of Cold Fusion, Proceedings of the 3rd International Conference on Cold Fusion, Dec. 1993 Nagoya, Japan, edited by H. Ikegami, Universal Academy Press, Tokyo, p. 21.

12. J. O'M Bockris, D. Hodko and Z. Minevski, "The mechanism of Deuterium Evolution on Palladium: Relation to Heat Bursts Provoked by Fluxing Deuterium Across the Interface" The Science of Cold Fusion, Proc. of the Second Annual Conference on Cold Fusion, Como, Italy, June 29-July 4, 1991, edited by T. Bressani, E. Del Giudice and G. Preparata, Published by Societa Italiana di Fisica, p. 337.

13. M. C. H. McKubre, S. Crouch-Baker, F. L. Tanzella, S. I. Smedley, M. Williams, S. Wing, M. Maly-Schreiber, R. C. Rocha-Filho, P. C. Searson, J. G. Pronko, and D. A. Koehler, Development of Advanced Concepts for Nuclear Processes in Deuterated Metals, EPRI Report TR-104195, August 1994.

14. J. O'M Bockris, R. Sundaresan, D. Letts and Z. Minevski, "Triggering of Heat and SubSurface Changes in Pd-D Systems" Proceedings of the 4th International Conference on Cold Fusion, Dec. 1993 Maui, Hawaii, edited by T. O. Passell and M. C. H. McKubre, Vol. 2, p. $1-1$.

15. R. Bass, "Proposed Nuclear Physics Experiment to Conclusively Demonstrate and Explain Aneutronic Cold Fusion," ICCF4 Proceedings, EPRI TR-104188-V4 (1994). 